\title{
Study on communication channel estimation by improved SOMP based on distributed compressed sensing
}

\author{
Biao Wang ${ }^{*}$, Yufeng Ge, Cheng He, You Wu and Zhiyu Zhu
}

\begin{abstract}
Wireless communication channel usually show the feature of time-varying; however, the time-varying channel has the characteristic that the channel structure within the adjacent time slots having serious time correlation. Therefore, how to use the time slow-changing characteristics of the channel to design the suitable channel state information acquisition method is of great significance to further improve communication performance with low communication bit error rate (BER) for OFDM communication system. The distributed compressed sensing (DCS) is proposed for the phenomenon that multiple sparse signals with time correlation. Based on the DCS theory framework, this article will re-build a time-domain channel estimation method with joint structure by improving the synchronous orthogonal matching pursuit (simultaneous orthogonal matching pursuit, SOMP) algorithm to get better channel information acquisition performance. Simulation results demonstrate the effectiveness of the proposed channel estimation method. Compared with the conventional compressed sensing-based channel estimations which perform at each time separately, the method proposed has better performance in terms of BER.
\end{abstract}

Keywords: Channel estimation, Compressed sensing, Joint sparse model, Orthogonal frequency division multiplexing (OFDM)

\section{Introduction}

Compressive sensing (CS) [1-3] as a new signal processing theory, when the signal is sparse in an orthogonal transformation domain, it can be under-sampled at a frequency far lower than Nyquist sampling rate, then the original sparse signal is recovered by nonlinear reconstruction algorithm with high probability. CS theory provides a new solution for traditional channel estimation, which has been applied to the channel estimation of a communication system by many scholars. Cotter and Rao proposed sparse channel estimation based on matching pursuit (MP) algorithm [4] for single-carrier communication system [5, 6]. Dongming and Bing extended the above sparse channel estimation method based on MP to the MIMO-OFDM multicarrier communication system [7]. Based on literature [8], C. R, Berger, S. Zhou, and others deeply studied the sparse channel estimation method based on CS, proposed an OFDM frequency domain channel estimation algorithm

\footnotetext{
* Correspondence: mail-wb@163.com

School of Electronic and Information, Jiangsu University of Science and Technology, Zhenjiang 212003, China
}

(c) The Author(s). 2019 Open Access This article is distributed under the terms of the Creative Commons Attribution 4.0 International License (http://creativecommons.org/licenses/by/4.0/), which permits unrestricted use, distribution, and reproduction in any medium, provided you give appropriate credit to the original author(s) and the source, provide a link to the Creative Commons license, and indicate if changes were made.

and analyzed the advantages and disadvantages of various sparse reconstruction algorithm, the simulation and experiment show that the bit error rate of BP channel estimation algorithm can reach lower bit error rate, which is far less than that of LS channel estimation [9]. In addition, the team mentioned above also conducted other CS-based channel estimation studies in 2010 and 2011, which all verified the superiority of CS-based algorithm over traditional algorithm [10, 11]. Compared with the traditional pilot aided method, CS-based method greatly reduces the number of insertion pilot frequencies and improves the communication efficiency when the performance of the channel estimation remains unchanged. But in the study of the CS theory applied to the channel, the CS reconstruction algorithm is based on the static sparse signal, only considering the sparse reconstruction of the single observable signal at a certain point of time. However, the high-speed mobile communication channel is often regarded as a coherent multipath channel with slow time-varying, the traditional channel estimation method based on static CS will seriously reduce the efficiency of channel estimation.

\section{Springer Open}

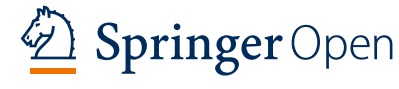


At present, some scholars have begun to study the application of compressed sensing for the processing of time-varying sparse signals. The existing research results show that for the dynamic characteristics of time-varying sparse signals, if the random signal processing method is used to conduct a dynamic reconstruction, a better sparse reconstruction effect will be obtained.

To sum up, the traditional channel estimation method has the disadvantages of high pilot cost and low spectral efficiency, the classic static CS channel estimation method has good performance of channel estimation, but due to the high complexity of the calculations, especially poor performance for tine-varying channel estimation. In this paper, based on this background, considering high-speed mobile communication channel is time-varying sparse and statistical dynamic, therefore, based on OFDM technology as the basic framework, a dynamic CS channel estimation method for high-speed vehicular communication channels is studied to further improve the performance of channel estimation and reduce the computational complexity of the algorithm.

\section{Methodology}

For DCS theory, the most typical application scenario is that multiple sensor nodes observe signals at the same time. These observed signals are usually non-sparse, but can be sparse represented under some sparse basis. More importantly, there is a certain correlation between the signals. When the corresponding signal is independently observed by each sensor, the original signal set can be recovered according to the observation results of each sensor node, and the reconstruction effect is better than that of each sensor node. Thus, it can be seen that the research object of DCS theory is the signal set with joint sparse characteristics. By analyzing the correlation structure between each sparse signal and adopting the strategy of joint signal recovery, the better sparse signal reconstruction effect is obtained.

This section will focus on JSM-1 and JSM-2 models commonly used in joint sparsity models (JSM):

\section{(a) JSM-1}

In the JSM-1 model, each signal in the observed signal set is composed of a common sparse part and a separate sparse part. Among them, all the signals contain a common sparse part, and the separate sparse part in each are not identical, meanwhile, the common sparse part and separate sparse part can be sparsely represented through the same sparse basis. The following is the mathematical description of this model:

$$
\mathbf{X}_{j}=\mathbf{Z}_{c}+\mathbf{Z}_{j}, \quad j \in\{1,2, \cdots, J\}
$$

Where $\mathbf{Z}_{c}=\boldsymbol{\Psi} \boldsymbol{\theta}_{c}$ represents the common sparse part of the signal, $\left\|\boldsymbol{\theta}_{c}\right\|_{0}=K_{c}$ represents $\mathbf{Z}_{c}$ is the sparse signal of $K_{c}, \mathbf{Z}_{j}=\boldsymbol{\Psi} \boldsymbol{\theta}_{j}$ represents the separate sparse part of the signal, and $\left\|\boldsymbol{\theta}_{j}\right\|_{0}=K_{j}$ represents $\mathbf{Z}_{j}$ is the sparse signal of $K_{j}$. In addition, the common sparse part only means that the coordinate of non-zero coefficient of the signal is the same, that is, the support set is the same.

(b) JSM-2

Unlike the JSM-1 model, all signals in the JSM-2 model have the same sparse support set but different non-zero coefficients. In this model, all the original signals can be constructed by the same sparse basis, and the mathematical representation is as follows:

$$
\mathbf{X}_{j}=\boldsymbol{\Psi} \boldsymbol{\theta}_{j}, \quad j \in\{1,2, \cdots, J\}
$$

Where $\left\|\boldsymbol{\theta}_{j}\right\|_{0}=K$ denotes that the sparsity of all signals is $K$. For the JSM-2 model, its typical application scenarios include MIMO communication, sound localization, etc. SOMP algorithm is also based on this model.

\subsection{The system model}

The OFDM channel estimation method based on CS is to transform the problem of OFDM channel estimation into the problem of sparse signal reconstruction, which is solved by the CS reconstruction algorithm. Specifically expressed as

$$
\mathbf{Y}_{P}=\mathbf{X}_{P} \mathbf{F}_{P} \mathbf{h}+\mathbf{W}_{P}=\mathbf{A h}+\mathbf{W}_{P}
$$

In the above equation, for the receiver, $\mathbf{Y}_{P} \mathbf{X}_{P} \mathbf{F}_{P}$ are all known and $h$ satisfies the sparse characteristic. Therefore, it is a typical CS theoretical model, which can reconstruct the time-domain channel response $h$ by using the $l_{1}$ minimum norm method or greedy algorithm with high probability.

On the other hand, high-speed mobile communication channel is considered to be slowly time-varying, and the channel coherence time is greater than the symbol period of OFDM. Within each OFDM symbol, the sparse multipath structure of communication channels remains unchanged. Therefore, the channel estimation method based on CS above is often carried out in a way of symbol-bysymbol, that is, the guide frequency is inserted into each OFDM symbol, and the impulse response of the channel is estimated by the receiver symbol-by-symbol. Although this method is simple and practical, it does not take into account the slowly varying characteristics of the high-speed mobile communication channel. Due to the channel change rate is slower than the OFDM symbol rate, channel impulse response has strong correlation within the duration of several consecutive OFDM symbols, namely, the corresponding channel response of each symbol have a common sparse part. The performance of channel estimation in OFDM system can be further improved if the 
characteristic of common sparse of channel response at each time can be fully utilized.

Based on this, the relevant literature, under the framework of DCS theory, converts the channel estimation under the slow time-varying channel into the problem of joint sparse recovery under the JSM-2 model, and carries out the joint sparse recovery through the SOMP algorithm to obtain channel response, which is introduced as follows:

According to Eq. (3), if the channel estimation within the time of continuous T OFDM symbols is taken into account, then

$$
\left\{\begin{array}{c}
\mathbf{Y}_{P}^{(1)}=\mathbf{A} \mathbf{h}^{(1)}+\mathbf{W}_{P}^{(1)} \\
\mathbf{Y}_{P}^{(2)}=\mathbf{A} \mathbf{h}^{(2)}+\mathbf{W}_{P}^{(2)} \\
\vdots \\
\mathbf{Y}_{P}^{(T)}=\mathbf{A} \mathbf{h}^{(T)}+\mathbf{W}_{P}^{(T)}
\end{array}\right.
$$

Where $\mathbf{Y}_{P}^{(t)}, \mathbf{h}^{(t)}, \mathbf{W}_{P}^{(t)}$ respectively represents the receiving signal at the pilot frequency, the impulse response of the time domain channel and the noise in the frequency domain of the $t$ th consecutive OFDM symbol, $1 \leq t \leq T$. When the channel changes slowly, the channel response $\mathbf{h}^{(t)}$ corresponding to $T$ consecutive OFDM symbols owns time correlation and common sparsity. By making full use of this common sparsity between signals, the channel estimation of multiple consecutive symbols can be considered as a whole, then the channel response $\mathbf{h}^{(t)}$ can be restored by joint sparse reconstruction instead of independent channel estimation of single OFDM symbol.

For the joint sparse model in Eq. (4), the following problems of optimization are constructed to solve the joint channel estimation:

$$
\hat{\mathbf{H}}=\arg \min \sum_{t=1}^{T}\left\|\mathbf{h}^{(t)}\right\|_{1} \quad \text { s.t. } \quad \sum_{t=1}^{T}\left\|\mathbf{Y}_{P}^{(t)}-\mathbf{A} \mathbf{h}^{(t)}\right\|_{2}^{2} \leq \varepsilon
$$

In Eq. (5), $\varepsilon$ is the parameter related to noise. The optimization problem above can be obtained by a joint reconstruction algorithm corresponding to JSM-2 model. This paper mainly discusses SOMP algorithm.

\subsection{Time domain joint channel estimation based on SOMP algorithm}

Signal sets constructed by different JSM models require different joint recovery algorithms to recover the signal. SOMP algorithm is a classic joint recovery algorithm in JSM-2 model, which was proposed by Tropp and Gilbert and then introduced into DCS environment by Baron [12]. As an improved greedy algorithm based on OMP algorithm, SOMP algorithm selects the atoms that best matched with the residual error to update the support set in each iteration and completes joint recovery of the signal set through multiple rapid iterations. However, the biggest difference between SOMP algorithm and OMP algorithm is that OMP algorithm is to select the atomic set that best matches the residual error of a single signal, while SOMP algorithm selects the atomic set that best matches the residual error of the whole signal group.

Combined with the basic principles of SOMP algorithm and the OFDM channel estimation model derived above, the time domain joint channel estimation based on SOMP algorithm is given. The specific steps are as follows:

1. Input: receive pilot signal $\mathbf{Y}_{P}=\left[\mathbf{Y}_{P}^{(1)}, \mathbf{Y}_{P}^{(2)}, \cdots\right.$, $\left.\mathbf{Y}_{P}{ }^{(T)}\right]$; the perception matrix $\boldsymbol{A}$; maximum sparsity $K$ of high-speed mobile communication channel.

2. Initialization: make the signal residual $\mathbf{r}_{0}^{(i)}=\mathbf{Y}^{(i)}, i$ $=1,2, \cdots, T$, the upper label represents the $i$ th OFDM symbol, and the lower index represents the number of iterations. The index set $\Lambda_{0}=$; reconstructed atomic set $\Phi_{0}=$; number of iterations $t=1$.

3. Iteration process: the th iteration. Step 1: Take the inner product of the perceived matrix $A$ and the residual $\mathbf{r}_{t-1}^{(i)}$, and find the inner product sum corresponding to T OFDM symbols, then calculate the maximum position $\lambda_{t}$ of the inner product sum:

$\lambda_{t}=\arg \max _{j} \sum_{i=1}^{T}\left|\left\langle\mathbf{A}_{j}, \mathbf{r}_{t-1}^{(i)}\right\rangle\right|$

Where $\mathbf{A}_{j}$ represents the $j$ th column atom of the perceptive matrix $A$;

Step 2: Update the index set and the reconstructed atomic set:

$\Lambda_{t}=\Lambda_{t-1} \cup \lambda_{t}$ $\Phi_{t}=\Phi_{t-1} \cup \mathbf{A}_{\lambda_{t}}$

Step 3: Using LS algorithm to calculate the multipath coefficient corresponding to each symbol:

$\hat{\mathbf{h}}_{t}^{(i)}=\arg \min \left\|\mathbf{Y}_{P}^{(i)}-\boldsymbol{\Phi}_{t} \mathbf{h}^{(i)}\right\|_{2}, i=1,2, \cdots, T$

Step 4: Update the residual according to the estimated value of channel impulse response:

$\mathbf{r}_{t}^{(i)}=\mathbf{Y}_{P}^{(i)}-\boldsymbol{\Phi}_{t} \hat{\mathbf{h}}_{t}^{(i)}, i=1,2, \cdots, T$

Step 5: Judge whether the condition $t=K$ is satisfied. If it satisfied, then stop iteration. If not, let $t=t+1$, execute step 1 .

4. Output: channel impulse response estimated value $\hat{\mathbf{h}}=\left[\hat{\mathbf{h}}^{(1)}, \hat{\mathbf{h}}^{(2)}, \cdots, \hat{\mathbf{h}}^{(T)}\right]$ corresponding to each OFDN symbol. 


\subsection{Analysis on the disadvantages of SOMP algorithm}

For the phenomenon that multiple sparse signals are correlated in distribution, DCS theory can further improve the recovery performance by utilizing the common sparsity between signals on the basis of classical CS theory, which has been widely studied and applied in wireless sensor network and MIMO communication.

However, the path delay of the actual high-speed mobile communication channel may change in multiple OFDM data symbols, and even the path generation and death may occur. The above time domain joint channel estimation based on SOMP algorithm assumes that the channel shares the same path time delay set in several continuous data symbols, which is obviously difficult to be completely satisfied and inconsistent with the actual situation! In addition, from the above description of the channel time correlation, it can be seen that the support sets for the channel sparse paths in adjacent OFDM symbols are not identical, in other words, there is a separate sparse multiple paths between the symbols, and therefore the JSM-1 model is clearly more compliant with the characteristics of the slowly varying high-speed mobile communication channel.

\subsection{OFDM channel estimation based on improved SOMP algorithm}

Through the analysis of time correlation of slow timevarying high-speed mobile communication channel, it can be seen that the JSM-1 model in DCS theory is more consistent with the characteristics of high-speed mobile communication channel than the JSM-2 model. In the JSM-1 model, each signal is composed of the common sparse part and the separate sparse part, so we can divide the channel path delay into two different parts, namely, the common channel tap and the dynamic channel tap. On the basis of this separation, an improved SOMP algorithm is proposed in this paper to reconstruct a time-varying sparse channel, the key idea of the proposed algorithm is to first simultaneously detect the common channel tap of the sparse channel in all OFDM data symbols, and then to use the path of the common channel tap as the initialization set in the dynamic channel tap tracking procedure, in order to track the dynamic channel tap and eliminate the wrong tap of the initialization set.

Combined with the symbol-by-symbol OFDM channel estimation model, the OFDM channel estimation process based on the improved SOMP algorithm is presented. The steps are as follows:

1. Input: receive pilot signal $\mathbf{Y}_{P}=\left[\mathbf{Y}_{P}^{(1)}, \mathbf{Y}_{P}{ }^{(2)}, \cdots\right.$, $\left.\mathbf{Y}_{P}{ }^{(T)}\right]$; the perception matrix $\boldsymbol{A}$; maximum sparsity $K$ of high-speed mobile communication channel.
2. Part 1: detection of common channel tap

$$
\Lambda_{0}=\operatorname{SOMP}\left(\mathbf{Y}_{P}, \mathbf{A}, K\right)
$$

3. Part 2: detection of dynamic channel tap Initialization: the index set $\Gamma_{0}^{(i)}=\Lambda_{0}$; reconstructed atomic set $\Phi_{0}^{(i)}=\mathbf{A}_{\Lambda_{0}}$; channel impulse response $\hat{\mathbf{h}}_{0}^{(i)}=\mathbf{A}_{\Lambda_{0}}^{\dagger} \mathbf{Y}_{P}^{(i)}$; residual $\mathbf{r}_{0}^{(i)}=\mathbf{Y}_{P}^{(i)}-\Phi_{0}^{(i)} \hat{\mathbf{h}}_{0}^{(i)}$; number of iterations $t=1$. In all the above symbols, the superscript represents the $i$ th OFDM symbol, and the subscript represents the number of iterations. Iteration process: the $t$ th iteration.

Step 1: Find out the column index $\lambda_{t}$ corresponding to the atom with the greatest correlation between residual error $\mathbf{r}_{t-1}^{(i)}$ and the perception matrix $A$, and update the index set and reconstructed atomic set.;

$$
\begin{gathered}
\lambda_{t}^{(i)}=\arg \max _{j}\left|\left\langle\mathbf{A}_{j}, \mathbf{r}_{t-1}^{(i)}\right\rangle\right| \\
\Gamma_{t}^{(i)}=\Gamma_{t-1}^{(i)} \cup \lambda_{t}^{(i)} \\
\Phi_{t}^{(i)}=\Phi_{t-1}^{(i)} \cup \mathbf{A}_{\lambda_{t}^{(i)}}
\end{gathered}
$$

Step 2: Using the LS algorithm to find the channel response in each symbol;

$\hat{\mathbf{h}}_{t}^{(i)}=\arg \min \left\|\mathbf{Y}_{P}^{(i)}-\Phi_{t}^{(i)} \mathbf{h}^{(i)}\right\|_{2}$

Step 3: Retain only the largest $K$ coefficients in $\hat{\mathbf{h}}_{t}^{(i)}$, and update the index set $\Gamma_{t}^{(i)}$ and reconstructed atomic set $\Phi_{t}^{(i)}$, use the LS algorithm to recalculate $\hat{\mathbf{h}}_{t}^{(i)}$;

Step 4: Update the residual:

$\mathbf{r}_{t}^{(i)}=Y_{P}^{(i)}-\Phi_{t}^{(i)} \hat{\mathbf{h}}_{t}^{(i)}$

Step 5: Judging whether the condition $\left\|\mathbf{r}_{t}^{(i)}\right\|_{2}$ $<\left\|\mathbf{r}_{t-1}^{(i)}\right\|_{2}$ is satisfied. If so, let $t=t+1$ then return to step 1. Otherwise $\Gamma_{t}^{(i)}=\Gamma_{t-1}^{(i)}, \hat{\mathbf{h}}_{t}^{(i)}=\arg \min$ $\left\|\mathbf{Y}_{P}^{(i)}-\Phi_{t}^{(i)} \mathbf{h}^{(i)}\right\|_{2}$, the iteration process ends.

4. Output: channel impulse response $\hat{\mathbf{h}}=\left[\hat{\mathbf{h}}^{(1)}, \hat{\mathbf{h}}^{(2)}, \cdots\right.$, $\left.\hat{\mathbf{h}}^{(T)}\right]$

The core steps of improved SOMP algorithm include two parts: common channel tap detection and dynamic channel tap tracking. The first part of the common channel tap detection is intended to estimate the common path delay set $\Lambda_{0}$ of each symbol, which is considered to contain the most real common channel taps. In this algorithm, this step is completed by the classical SOMP algorithm. After this, the time delay set $\Lambda_{0}$ is used as the initial support set for dynamic channel tap tracking within each symbol. 
In the second part of the algorithm, the dynamic channel tap tracking is designed to track the different time-varying channel taps within different OFDM symbols, namely, the separate sparse part in the JSM-1 model. Within each symbol, the common path delay set $\Lambda_{0}$ obtained in the first part of the algorithm is used to initiate the dynamic tracking process. Through each iteration, the size $K$ of the path delay set remains unchanged and only the reconstruction effect is enhanced. In addition, reliable or incorrect channel taps can be added or removed from the path delay set during each iteration. Benefit from the detection of the common channel tap, the dynamic channel tap tracking process can rapidly approach the real path delay set, and when the residual error signal $\mathbf{r}_{t}^{(i)}$ is no longer reduced, namely, $\left\|\mathbf{r}_{t}^{(i)}\right\|_{2}<\left\|\mathbf{r}_{t-1}^{(i)}\right\|_{2}$, the process will be terminated.

\section{Simulation results and discussion}

\subsection{Parameter settings}

In order to verify the effectiveness of the algorithm, the proposed channel estimation based on the improved SOMP algorithm is simulated and analyzed, and the proposed algorithm is compared with other similar algorithms in this section. In the simulation system, the total number of subcarriers of OFDM system is set to 256, the protection interval length is 64 cyclic prefixes, and the number of pilot frequencies inserted by a single OFDM symbol is 32 pilot frequencies according to the data. The pilot insertion mode is determined according to the channel estimation method. The conventional LS channel estimation adopts comb pilot insertion with equal interval, but the channel estimation based on CS adopts random pilot insertion. All modulation modes adopt QPSK modulation, and there is no channel coding, the receiver assumes full synchronization.

Because the proposed algorithm is based on the JSM-1 model, the sparse multipath setting in channel simulation includes the common sparse multipath and dynamic sparse multipath. Where the common sparse multipath exists within each symbol, the delay position remains unchanged, only the amplitude changes with time, and the dynamic sparse multipath within each symbol is generated randomly. All sparse multipath are independent of each other, and the gain follows the complex Gaussian distribution of zero mean and decays exponentially. Assuming that the channel length $L=60$, each multipath time delay is an integer multiple of the system sampling interval without energy leakage. Under the condition of high-speed mobile communication channel, the channel state remains unchanged within an OFDM symbol period, and each OFDM symbol is independent of each other.

\subsection{Results discussion}

Based on the above system simulation parameters, the following sets of the simulation are made in this paper: (a) The effect of the number of common channel tap on the performance of the channel estimation algorithm

Since the proposed algorithm is based on the JSM-1 model, the proportion of common and separate sparse paths in the channel will influence the channel estimation algorithm. When the number of common sparse multipath increases, the correlation of the channel multipath sparse structure within the adjacent OFDM symbol is enhanced, and vice versa. In this simulation, the maximum sparsity $K$ of the channel is 10 , and the number of the public channel taps is represented by the time correlation $L$. The specific value varies from 2 to 8 . The simulation results are shown in Fig. 1. In addition to the algorithm mentioned in this paper, LS, OMP, SOMP, and Oracle-LS algorithms are also used as comparison algorithms. It can be seen from Fig. 1 that SOMP algorithm is the one most affected by time correlation $L$ of all algorithms, and the other algorithms have no significant influence. Because the proposed algorithm includes dynamic channel tap detection, the change of $\mathrm{L}$ only affects the computational complexity of the algorithm, but not the performance of the algorithm. As for the SOMP algorithm, it shares the same path delay set by default, so when $L$ increases, the same path delay number increases, the algorithm performance improves. On the contrary, the performance of the algorithm reduces. As can be seen from the figure, when $L=K=10$, the channel degenerates into jsm2 model. At this point, the algorithm proposed in this paper also degenerates into SOMP algorithm, so the estimated performance of the two algorithms is equivalent.

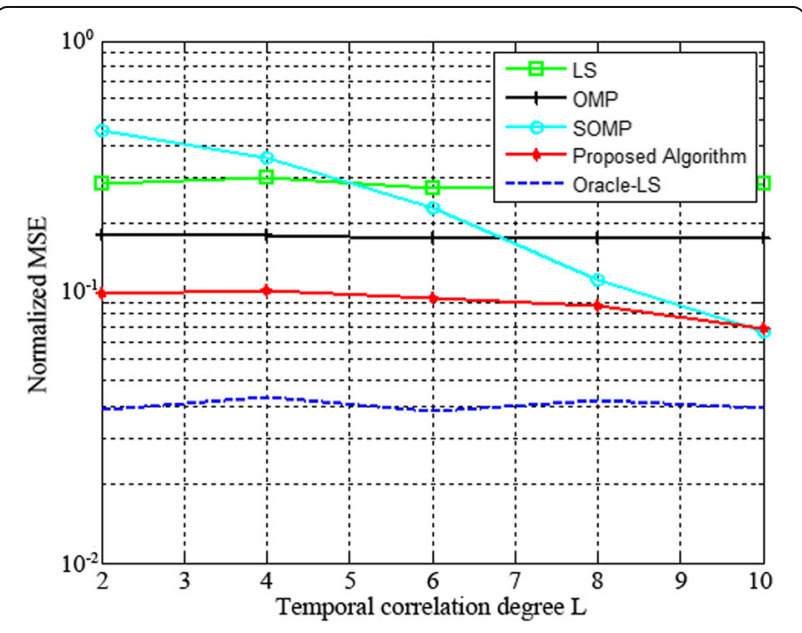

Fig. 1 The NMSE with different temporal correlation degree $L$ 
(b) The effect of the number of joint symbol on detection of common channel tap.

As an improved algorithm of SOMP algorithm, the proposed algorithm is also based on joint sparse model. As can be seen from the description of the algorithm above, detection of common channel tap in the first part of the algorithm is to use SOMP algorithm to combine multiple OFDM symbols. Because the delay set of the common channel tap will be the initial support set of the dynamic channel tap detection in the second part, the success rate of the common channel tap detection directly affects the performance and speed of the second part of the algorithm. Figure 2 below simulates the effect of four different joint symbol numbers on the success rate of common channel tap detection. It is not hard to find that the bigger the number of joint symbols, the higher the detection success rate of common channel tap. For this phenomenon, it can be explained that the public sparse multipath exists at all times. When combined with SOMP algorithm recovery, the energy of the weak path will be enhanced by superimposing the same path in multiple symbols, so that it can be detected more easily. If the number of symbols involved in the superposition increases, the detected probability will also increase.

(c) NMSE and BER curves of various algorithms with different SNR.

The channel estimation performances of the proposed algorithm in this paper are compared in this group of simulation. The normalized mean square error and error rate are adopted for the specific evaluation indexes. In this paper,

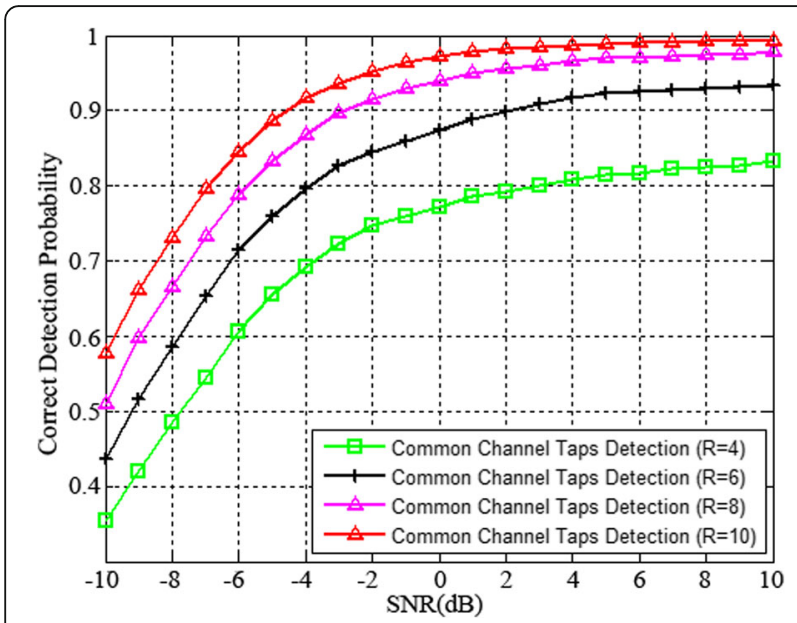

Fig. 2 Correct detection probability of common channel taps with different SNR normalized mean square error (NMSE) is defined as follows:

NMSE $=\frac{1}{T} \sum_{t=1}^{T} \frac{\left\|\hat{\mathbf{h}}^{(t)}-\mathbf{h}^{(t)}\right\|_{2}^{2}}{\left\|\mathbf{h}^{(t)}\right\|_{2}^{2}}$

In Eq. (14), $\mathbf{h}^{(t)}$ represents the impulse response of the ideal channel, and $\hat{\mathbf{h}}^{(t)}$ represents the channel estimated value obtained by various algorithms. The simulation results of NMSE and BER are shown in Fig. 3 and Fig. 4 respectively. In these two simulations, the number of common channel taps and the maximum sparsity of the channel are set as $L=8$ and $K=10$ respectively. The channel estimation algorithms involved in the comparison include LS, OMP, SOMP, and Oracle-LS algorithms. In order to ensure the performance of LS algorithm, the pilot insertion mode is comb pilot with uniform spacing of 8 , and the rest of CS algorithms adopt random pilot. It is not hard to find from Fig. 3 and Fig. 4 that the performance curves of NMSE and BER tend to be basically the same. Among them, LS algorithm, as a traditional algorithm, has the worst estimation performance because the pilot frequency is smaller than the channel length and the algorithm itself is greatly affected by noise when estimating the sparse path. For CS-based channel estimation, SOMP algorithm has a great advantage over OMP algorithm when SNR is low. The reason is that when SNR is low, SOMP algorithm can combine multiple symbols for channel reconstruction, and its detection ability of weak path is better than that of OMP algorithm which considers only one symbol. When SNR increases, due to the inherent defects of SOMP

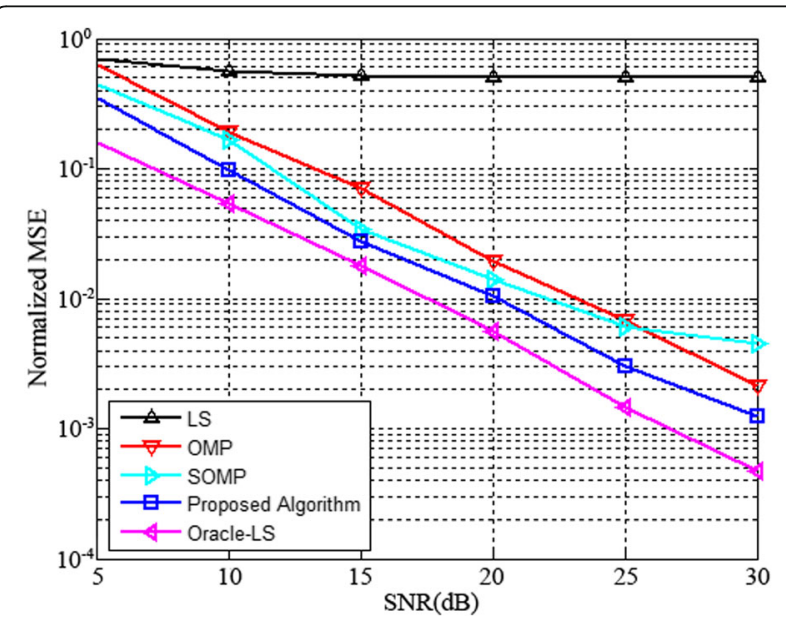

Fig. 3 The NMSE with different SNR 


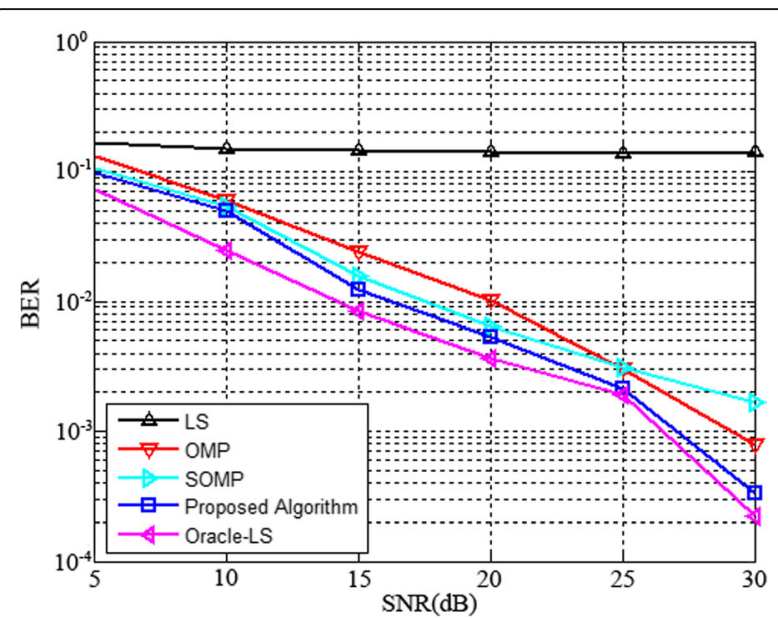

Fig. 4 The BER with different SNR

algorithm model in time-varying channel, the performance improvement effect is limited. OMP algorithm is less affected by noise in high SNR environment, every time the reconstructed channel can be completed $\mathrm{K}$ iterations, so the performance is better under high SNR environment. As for the improved SOMP algorithm proposed in this paper, it is better than OMP and SOMP algorithm because it considers the detection of dynamic channel tap, which is consistent with the results of the previous mathematical analysis. In addition, the comparison algorithm Oracle-LS in the simulation of this group is the LS estimation when the multipath delay set of time-varying sparse channel is known, and this result is the theoretical limit value under the LS criterion.

(d) Analysis of algorithm complexity under different channel sparsity.

For greedy algorithm, the number of iterations is generally signal sparsity $K$. The higher the $K$ value of signal sparsity is, the higher the complexity of greedy algorithm. The proposed algorithm is also a greedy algorithm, so the complexity of the algorithm is analyzed by calculating the CPU running time of channels estimation with different $K$ sparsity. In the simulation, the estimated channel time of 10 consecutive OFDM symbols is taken as CPU running time, a total of 100 simulations were performed. The comparison algorithm of this group of the simulation consists of LS, OMP, SP, and SOMP algorithms. The simulation results are shown in Fig. 5. As it can be seen from Fig. 5, except the LS algorithm, the $\mathrm{CPU}$ running time of the other algorithms increases exponentially with the increase of $K$ value. Among the four greedy algorithms, the OMP channel estimation and the SP channel estimation are performed symbol by symbol,

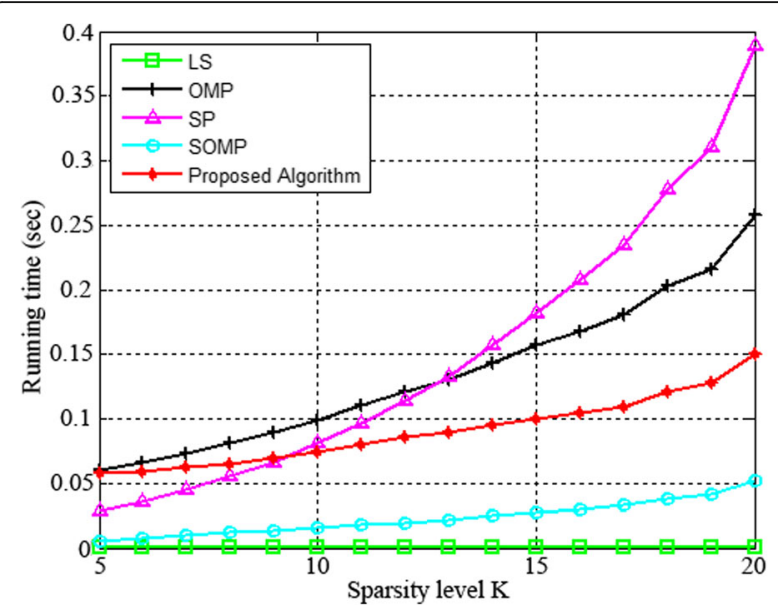

Fig. 5 CPU running time with different $K$ sparse channels

so it is much larger in time than the channel estimation algorithm based on the joint sparse model. Particularly, the SP algorithm, because each iteration takes $K$ column atoms, so when $K$ increases, the algorithm complexity also has a rapidly increase, which is most obvious in all the algorithms. Among the SOMP algorithm and the proposed algorithm in this paper, due to the improved SOMP algorithm increases the dynamic channel tap detection, so the CPU running time is longer, which is equivalent to sacrificing speed for higher estimation accuracy.

\section{Conclusion}

This paper mainly studies the joint estimation method of high-speed mobile communication channel based on improved SOMP algorithm. Firstly, the distributed compressed sensing theory and joint sparse model are introduced. Secondly, the existing time domain joint channel estimation method based on SOMP algorithm is introduced. On this basis, this paper analyzes the defects of existing algorithms that do not take into account channel time-varying and proposes an improved SOMP algorithm based on JSM1 model for recovery of joint channel estimation. Simulation results show that the proposed algorithm in this paper has greater advantages of estimation performance and lower algorithm complexity compared with the existing symbol-by-symbol CS channel estimation algorithm in the time-varying channel environment.

\section{Abbreviations}

BER: Bit error rate; CS: Compressive sensing; DCS: Distributed compressed sensing; SOMP: Simultaneous orthogonal matching pursuit

\section{Acknowledgements}

The research presented in this paper was supported by National Natural Science Foundation of China, Natural Science Foundation of Jiangsu

Province of China. 


\section{Funding}

The authors acknowledge the National Natural Science Foundation of China (grant 11574120, U1636117), the Natural Science Foundation of Jiangsu Province of China (grant BK20161359), The Science and Technology on Underwater Acoustic Antagonizing Laboratory, Systems Engineering Research Institute of CSSC (grant MB80038).

\section{Availability of data and materials}

Not applicable.

\section{Authors' contributions}

YG and BW are the main writers of this paper. They proposed the main idea, deduced the algorithm theory, completed the simulation, and analyzed the result. CH and YW give some work for simulation. ZZ gave some important suggestions for improving the SOMP. All authors read and approved the final manuscript

\section{Competing interests}

The authors declare that they have no competing interests.

\section{Publisher's Note}

Springer Nature remains neutral with regard to jurisdictional claims in published maps and institutional affiliations.

Received: 22 February 2019 Accepted: 3 May 2019

Published online: 17 May 2019

\section{References}

1. D.L. Donoho, Compressed sensing [J]. IEEE Trans. Inf. Theory 52(4), 12891306 (2006)

2. E.J. Candès, J. Romberg, T. Tao, Robust uncertainty principles: Exact signal reconstruction from highly incomplete frequency information []]. IEEE Trans. Inf. Theory 52(2), 489-509 (2006)

3. E.J. Candès, M.B. Wakin, An introduction to compressive sampling [J]. IEEE Signal Process. Mag. 25(2), 21-30 (2008)

4. S.G. Mallat, Z. Zhang, Matching pursuits with time-frequency dictionaries [J]. IEEE Trans. Signal Process. 41(12), 3397-3415 (1993)

5. S.F. Cotter, B.D. Rao, Matching pursuit based decision-feedback equalizers [C]// IEEE international conference on acoustics, speech. Signal Process., 2713-2716 (2000)

6. S.F. Cotter, B.D. Rao, Sparse channel estimation via matching pursuit with application to equalization [J]. IEEE Trans. Wirel. Commun. 50(3), 374-377 (2002)

7. Wang D, Han B, Zhao J, et al. Channel estimation algorithms for broadband MIMO-OFDM sparse channel [C]// IEEE 2003 international symposium on personal, Indoor and Mobile Radio Communications. 2003: 1929-1933

8. W. Li, J.C. Preisig, Estimation of rapidly time-varying sparse channels [J]. IEEE J. Ocean. Eng. 32(4), 927-939 (2008)

9. Mason S, Berger C, Zhou S, et al. An OFDM design for underwater acoustic channels with Doppler spread [C]// Digital Signal Processing Workshop and IEEE Signal Processing Education Workshop. 2009: 138-143

10. C.R. Berger, Z. Wang, J. Huang, et al., Application of compressive sensing to sparse channel estimation [J]. IEEE Commun. Mag. 48(11), 164-174 (2010)

11. J. Huang, S. Zhou, J. Huang, et al., Progressive inter-carrier interference equalization for OFDM transmission over time-varying underwater acoustic channels []]. IEEE Journal of Selected Topics in Signal Processing 5(8), 15241536 (2011)

12. J.A. Tropp; A.C. Gilbert; M.J. Strauss. Simultaneous sparse approximation via greedy pursuit [C].// proceedings. (ICASSP '05). IEEE international conference on acoustics, speech, and Signal Process., 2005:721-724

\section{Submit your manuscript to a SpringerOpen ${ }^{\circ}$ journal and benefit from:}

- Convenient online submission

- Rigorous peer review

- Open access: articles freely available online

High visibility within the field

Retaining the copyright to your article

Submit your next manuscript at $\boldsymbol{\nabla}$ springeropen.com 